\title{
A PSICOLOGIA DO TRABALHO QUE REFLETE SOBRE SEU PRÓPRIO PERCURSO DE PESQUISA: O CASO DA INTERDIÇÃO DE ENTREVISTA DOS MÉDICOS CUBANOS ATUANDO NO PROGRAMA MAIS MÉDICOS - BRASIL
}

PSYCHOLOGY OF LABOR ACTIVITY REFLECTING UPON ITS OWN RESEARCH PATHWAY: THE CASE OF INTERDICTION FOR PARTICIPATING IN INTERVIEWS OF CUBAN MEDICAL DOCTORS ACTING IN MAIS MÉDICOS BRAZILIAN PROGRAM

LA PSICOLOGÍA DEL TRABAJO QUE REFLEXIONA SOBRE SU PROPIO CAMINO DE INVESTIGACIÓN: EL CASO DE PROHIBICIÓN DE ENTREVISTAS DE LOS MÉDICOS CUBANOS QUE ACTÚAN EN EL PROGRAMA MAIS MÉDICOS - BRASIL

\section{Jorge Tarcísio da Rocha Falcão* \\ Marie Baraud* \\ Nadja Acioly-Régnier ${ }^{* *}$}

\begin{abstract}
RESUMO
O presente artigo apresenta meta-análise de um fato real que, em princípio, apareceu para seus autores como um obstáculo à realização de projeto original de pesquisa, que passa, após refinamento de análise, à condição de parte constitutiva da pesquisa. Trata-se de impedimento à realização de entrevistas com médicos cubanos participantes do programa institucional brasileiro "Mais Médicos", no contexto de pesquisa voltada para a análise do impacto da mobilidade de profissionais médicos do país de origem para país estrangeiro. Tal impedimento foi imposto pela autoridade cubana de acompanhamento dos referidos médicos. Reflete-se aqui acerca do princípio segundo o qual eleger a abordagem psicossociológica como referencial epistemológico, teórico e metodológico implica tratar esse tipo de vicissitude como elemento constitutivo, e não impeditivo da pesquisa. Tal postura resulta de esforço crítico contínuo do pesquisador, para o qual o estabelecimento prévio de desenho de pesquisa e sua devida realização continua ainda a postura hegemônica.
\end{abstract}

Palavras-chave: Psicossociologia do trabalho. Médicos estrangeiros. Programa Mais Médicos. Teoria e método.

\footnotetext{
* Psicólogo, doutor em psicologia pela Universidade de Paris 5 (René Descartes), com estágio pós-doutoral junto ao grupo de pesquisa da Clinica da Atividade, liderado pelo prof. Yves Clot, no CNAM (Conservatoire National des Arts et Métiers), França. É professor titular e pesquisador do Departamento de Psicologia da Universidade Federal do Rio Grande do Norte (UFRN), pró-reitor adjunto de pesquisa desta universidade

** Université Lumière-Lyon 2, Laboratoire «Santé Individu Société». E-mail: marie.brd@gmail.com

***Université Lumière-Lyon 1, Laboratoire «Santé Individu Société». Email: acioly.regnier@wanadoo.fr
} 


\begin{abstract}
This paper aims to share a theoretical and methateoretical analysis about an unexpected refusal of subject's participation in a research, initially seen as an obstacle to the accomplishment of previous research design, this analysis being changed afterwards into constitutive aspect of the research subject itself. Cuban medical practionners integrated to Brazilian project "Mais Médicos" were forbidden by Cuban authorities to be interviewed by Brazilian researchers, in the context of a research about medical professional practice of workers acting in a foreign country. We demonstrate here that considering psychossociological approach of labor activity as a theoretical and methodological landmark implies in considering such "obstacles" of research design as a constitutive aspect of research. We also show, through a reflexive analysis, that the approach mentioned above is not at all easy, since traditional methodological attitude of previous establishment and strict respect of research design is always hegemonic.
\end{abstract}

Keywords: Psychosociology of work. Foreign medical practionners. Mais Médicos Program. Theory and method.

\title{
RESUMEN
}

El presente artículo presenta meta-análisis de hecho real que, en principio, apareció para sus autores como un obstáculo a la realización de proyecto original de investigación, que pasa, después del refinamiento de análisis, a la condición de parte constitutiva de la investigación. Se trata de impedimento a la realización de entrevistas con médicos cubanos participantes del programa institucional brasileño "Más Médicos", en el contexto de investigación orientada al análisis del impacto de la movilidad de profesionales médicos del país de origen para país extranjero. Este impedimento fue impuesto por la autoridad cubana de acompañamiento de dichos médicos. Se reflexiona aquí acerca del principio según el cual elegir el enfoque psicosociológico como referencial epistemológico, teórico y metodológico implica tratar ese tipo de vicisitud como elemento constitutivo, y no impeditivo de la investigación. Esta postura resulta de un esfuerzo crítico continuo del investigador, para el cual el establecimiento previo de diseño de investigación y su debida realización sigue siendo la postura hegemónica.

Palabras clave: Psicosociología del trabajo. Médicos extranjeros. Mais Médicos. Teoría y método.

\section{CONTEXTUALIZAÇÃO INICIAL E PROBLEMÁTICA}

presenta-se aqui reflexão sobre a amplitude dos aspectos de contextualização
da pesquisa que tem como quadro epistemológico, teórico e metodológico
a abordagem psicossociológica do trabalho. Trata-se de mostrar que, 
diferentemente de contextos metodológicos mais tradicionais de pesquisa, aspectos comumente considerados como "obstáculos" à realização dos projetos de pesquisa passam necessariamente a fazer parte da própria pesquisa. Não fosse assim, como considerar que o contexto social, histórico e cultural que enquadra toda manifestação humana tem necessariamente papel preponderante na pesquisa psicossociológica?

Em pesquisa de tese de doutoramento voltada para a abordagem da atividade profissional de médicos que exercem seu trabalho em contexto diverso daquele onde obtiveram sua formação profissional, buscou-se observar e contrastar (sem o intuito estrito da comparação) contextos de trabalho francês e brasileiro, no âmbito dos quais médicos estrangeiros (em relação a esses dois contextos nacionais) exercem a profissão (Baraud, 2016). Tal pesquisa buscou evidenciar e detalhar as transformações em termos de processos identitários decorrentes do exercício da profissão fora do país de obtenção do título de médico, abarcando, com esse intuito, grupo de médicos exercendo a profissão na França, originários do leste europeu (notadamente Romênia) e países árabes do norte da África (notadamente Argélia e Marrocos), e grupo de médicos exercendo a medicina no Brasil, a maioria absoluta dos quais proveniente de Cuba, no âmbito do programa federal Mais Médicos (Brasil, 2017).

Para a obtenção de dados necessários às análises conducentes à realização dos objetivos acima mencionados, previram-se distintas etapas de trabalho. Inicialmente, os participantes responderiam a questionário destinado a caracterizar, no âmbito do grupo, aspectos relacionados à motivação para a migração empreendida. Em seguida, previu-se a realização de entrevistas do tipo relato autobiográfico com pequenos efetivos $(n=8)$ oriundos dos grupos trabalhando na França e no Brasil, de forma a ensejar relatos acerca da trajetória pessoal, narrada a partir da perspectiva do próprio sujeito. Pretendeu-se, com essa etapa, enriquecer as informações oriundas da etapa anterior com depoimentos baseados em vivências ou perehzvanie (Veresov, 2014), ou seja, experiências do percurso biográfico de cada participante cujo relato abarcasse o registro de emoções e afetos vinculados ao vivido. Finalmente, previu-se etapa de análise documental, abarcando material divulgado na imprensa acerca das atividades dos médicos estrangeiros em seus países de recepção.

Mais do que trazer à discussão os elementos relacionados a tais fontes de dados, e os próprios dados obtidos, pretende-se aqui discutir reflexivamente sobre circunstância inesperada, representada pela não-autorização, por parte da autoridade cubana responsável pela supervisão das atividades profissionais dos médicos cubanos participantes do Programa Mais Médicos no Brasil - Organização Panamericana de Saúde (OPAS), para a participação de qualquer médico cubano 
trabalhando em território brasileiro na etapa de entrevista autobiográfica acima mencionada. Já na etapa de resposta aos questionários disponibilizados em endereço acessível na web, houve dificuldade em obter da OPAS e do Ministério da Saúde a lista de endereços eletrônicos dos médicos cubanos atuando nos diversos municípios brasileiros de recepção - dificuldade finalmente contornada, com o auxílio de docentes vinculados à Universidade Federal do Rio Grande do Norte (UFRN) e atuando na tutoria dos médicos cubanos exercendo a medicina no Estado do Rio Grande do Norte. Isso permitiu a obtenção de 46 questionários respondidos pelo grupo de cubanos (em complemento aos 43 recebidos do grupo de médicos atuando em território francês). Não obstante, não se conseguiu autorização para a realização das entrevistas autobiográficas, apesar de vários contatos diretos do diretor de tese e pró-reitor de pesquisa junto à UFRN. Os motivos alegados eram desconhecimento dos detalhes da pesquisa e, portanto, necessidade de tempo para o devido exame (resumos em português e espanhol foram encaminhados às referidas autoridades de tutela, sem que até o momento em que se redige o presente relato - que coincide com a época de defesa da tese de doutorado aqui referida - qualquer resposta, positiva ou negativa, tenha chegado).

\section{ABORDAGEM PSICOSSOCIOLÓGICA DA ATIVIDADE DE TRABALHO: MARCOS TEÓRICOS CENTRAIS}

O conjunto de abordagens em psicologia do trabalho que são abarcadas pelo qualificativo acima tem algumas características centrais e comuns (Bendassolli \& Sobol, 2010; Lhuilier, 2006). Talvez a mais central delas, que resume com mais propriedade esse núcleo duro de atributos comuns, seja a consideração de que a atividade de trabalho, sob essa ótica, não pode perder de vista os processos singulares e atravessados de emoção e afeto que acompanham o processo desenvolvimental de individuaçāo. Além disso, tal processo ocorre, simultaneamente, apesar e em oposição aos demais sujeitos (os "outros"), e em processo de co-gênese que necessariamente convoca esses "outros". Dito de outra forma, a individuação é um processo abordado em contexto de dialogicidade (Markovà, 2000, 2006; Rommetveit, 2003; Valsiner, 2000, 2014). Por conta dessa perspectiva, a psicologia do trabalho que eleja como marco teórico de enquadre a abordagem psicossociológica tem necessariamente, como unidade de análise, indivíduos em interação com outros indivíduos, no âmbito da necessidade de agir (Clot, 2010a), que demanda necessariamente a mediação de um contexto mais amplo que dê sentido e substância real a esse agir. Tal mediação será responsável por facilitaçôes do poder de agir individual, por ocasião da oferta de um excedente de visão por um parceiro (Bakhtine, 1977) ou empoderamento 
decorrente do acesso a amplificadores culturais (Bruner, 2014) ou por obliteração dificultadora, vivida pelo sujeito como impedimento a seu poder de agir.

A abordagem psicossociológica do contexto de trabalho ressalta que as competências profissionais lutam todo o tempo para se traduzirem em efeitos "úteis", ou seja, efeitos que se põem a serviço de objetivos que transcendem a esfera do indivíduo. Isso significa que a convicção pessoal do indivíduo trabalhador, no sentido de que ele, indivíduo, está realizando adequadamente seu trabalho, pode não coincidir com a avaliação da organização na qual exerce esse trabalho. É essa possibilidade que leva Yves Clot a distinguir "qualidade do trabalho" e "trabalho de qualidade" (Clot, 2010b, p. 37). Tal abordagem da atividade do trabalho, tem como unidade de análise não propriamente o indivíduo que é confrontado eventualmente a obstáculos oriundos do contexto real em que se insere, e sim o poder de agir desse indivíduo no contexto de trabalho, ou seja, a margem de que goza o indivíduo trabalhador para adaptar, otimizar, corrigir, "estilizar" sua prática profissional na direção de uma melhoria da mesma (Clot, 2010a). Nesse sentido, instâncias ligadas ao indivíduo, em sua subjetividade e saúde física e mental, poderão e deverão ser consideradas, mas em contexto de interação com instâncias do coletivo de trabalhadores, do gênero profissional, da organização à qual o indivíduo trabalhador se filia, das vicissitudes do dia-a-dia real da prática profissional do trabalhador. Obstáculos com os quais se defronta o indivíduo na realização de sua atividade de trabalho não são algo externo a essa atividade: constituem a atividade real - mesmo que não esgotem o amplo espectro de possíveis que são abarcados pelo real da atividade.

\section{ABORDAGEM PSICOSSOCIOLÓGICA DA ATIVIDADE DE TRABALHO: DESDOBRAMENTOS METODOLÓGICOS}

Os desdobramentos metodológicos da perspectiva acima conduzem à formulação de projetos de pesquisa que respeitem as especificidades do real, bem como o processo de co-gênese do indivíduo às voltas por se demarcar ou ultrapassar esse contexto limitador, sem dele se separar, no âmbito do que Valsiner (2001) denominou "separação inclusiva”. Disso decorre que projetos de pesquisa voltados para a descrição da atividade de trabalho precisam comportar margem de sujeição metodológica que os habilite a incorporar qualquer obstáculo à atividade de trabalho como elemento descritor dessa mesma atividade. Trazendo esse preceito para o contexto específico da pesquisa que ensejou a presente reflexão, o aspecto dificultador da plena realização do projeto de pesquisa, do qual resultou tese de doutoramento já concluída e em fase de defesa (Baraud, 2016), não poderia ser visto como elemento adverso que "impediu" a plena realização do projeto inicial, 
e sim como elemento de valor positivo sem o qual, doravante, não mais poderse-ia abordar adequadamente a descrição da atividade de trabalho dos médicos cubanos no contexto do programa institucional brasileiro Mais Médicos.

\section{A RECUSA DE AUTORIZAÇÃO DE ENTREVISTAS DOS MÉDICOS CUBANOS DO PROGRAMA MAIS MÉDICOS NO ÂMBITO DA DESCRIÇÃO DE SUA ATIVIDADE DE TRABALHO}

O impedimento da realização das entrevistas com os médicos cubanos, por parte de autoridade cubana de tutela em território brasileiro, forneceu subsídios que se mostraram valiosos para a presente pesquisa. Não há como eludir o fato de que a imposição da referida proibição representa afronta à liberdade desses médicos, não somente em sua atividade profissional, mas também no contexto da própria cidadania. Não obstante, para além do estranhamento, é preciso o recuo crítico que situe o obstáculo à pesquisa como parte integrante dessa mesma pesquisa. Os médicos cubanos participantes do Programa Mais Médicos, seja no Brasil ou em outros países, se comprometem a respeitar cláusula de confidencialidade a respeito de informações, normas e episódios relacionados à atividade profissional no âmbito do programa. Tal postura demanda naturalmente a consideração, em ordem ascendente de complexidade, de aspectos que vão desde a proposição inicial do programa, no que diz respeito à constituição de força-tarefa de médicos para expedições fora de Cuba, como tem sido o caso das missóes cubanas à África, no contexto do combate ao vírus Ebola (Walter, Pontes, \& Valle, 2014) até a consideração da natureza político-ideológica do regime cubano em sua feição atual (Brito, 2014). Há que se considerar, ainda, que o foco da presente pesquisa não eram as informações confidenciais do programa, mas a vivência (Veresov, 2014) e a experiência pessoal deles, no bojo de uma narrativa reflexiva sobre a trajetória migratória de cada um em contexto de globalização da prática da medicina. Não se pretende aqui inferir, de forma ingênua, que tais narrativas estivessem completamente isoladas das referidas informações sensíveis - provavelmente não, o que constitui argumento a favor da plausibilidade do interdito em questão. Mas, por outro lado, tais narrativas têm-se produzido, em contexto brasileiro, de forma espontânea e ocasionalmente em confronto aberto com as normas programáticas cubanas ${ }^{1}$. Alguns médicos participantes do programa até se ofereceram para participar das entrevistas, independentemente da autorização da autoridade de tutela.

O objetivo central das entrevistas, conforme aludido acima, era obter dados complementares para a investigação dos processos afetivos vinculados às transformações identitárias dos médicos em situação de mobilidade, exercendo

\footnotetext{
1 "Não somos prisioneiros do regime, dizem médicos cubanos ao chegar à Bahia" (Barros Neto, 2013).
} 
a medicina em país diverso daquele onde obtiveram sua formação e acreditação profissionais. O objetivo, em primeira instância, era, portanto, a co-construção de narrativas sobre as histórias pessoais deles. Nesse contexto, a não-autorização para a realização das entrevistas com o grupo de médicos cubanos contactados e já previamente de acordo em concedê-las permitiu o acesso a um aspecto da relação institucional entre esses médicos e a instância de tutela governamental cubana que talvez não se explicitasse dessa maneira no contexto das entrevistas. Essa situação permitiu refinar análises interpretativas a respeito do contexto de atuação profissional dos médicos cubanos no Programa Mais Médicos, do que resultou a reorientação do trabalho de análise documental que combinou fontes videográficas (Melgaço, 2014) e artigos de jornais da grande imprensa brasileira. Foram retomados principalmente dois fatos marcantes no percurso biográfico de todos eles: a privação da livre expressão e a impossibilidade de trazer de Cuba as respectivas famílias e morar com elas no Brasil. Cabe aqui considerar que a maior parte dos médicos desse grupo tem relação civil estável - casamento ou união livre (dados resumidos na Tabela 1).

Tabela 1. Perfil sociodemográfico dos médicos cubanos do grupo de participantes, exercendo a profissão no Brasil no contexto do Programa Mais Médicos

\begin{tabular}{ccc}
\hline VARIAVEIS SOCIODEMOGRAFICAS & EFETIVO & PORCENTAGEM \\
\hline Sexo & & \\
\hline Masculino & 17 & $36,2 \%$ \\
Feminino & 30 & $63,8 \%$
\end{tabular}

\begin{tabular}{ccc}
\hline Idade & & \\
\hline 30 & 2 & $4,3 \%$ \\
31 & 1 & $2,1 \%$ \\
32 & 2 & $4,3 \%$ \\
33 & 3 & $6,4 \%$ \\
34 & 1 & $2,1 \%$ \\
35 & 3 & $6,4 \%$ \\
36 & 2 & $4,3 \%$ \\
37 & 6 & $12,8 \%$ \\
38 & 1 & $2,1 \%$ \\
39 & 1 & $2,1 \%$ \\
40 & 1 & $2,1 \%$ \\
41 & 1 & $2,1 \%$ \\
43 & 3 & $6,4 \%$ \\
44 & 1 & $2,1 \%$ \\
45 & 2 & $4,3 \%$
\end{tabular}




\begin{tabular}{ccc}
46 & 1 & $2,1 \%$ \\
47 & 3 & $6,4 \%$ \\
48 & 3 & $6,4 \%$ \\
49 & 4 & $8,5 \%$ \\
50 & 2 & $4,3 \%$ \\
51 & 3 & $6,4 \%$ \\
52 & 1 & $2,1 \%$ \\
& & \\
Situação civil & & $25,5 \%$ \\
\hline Solteiro & 12 & $14,9 \%$ \\
União livre & 7 & $44,7 \%$ \\
Casado & 21 & $10,6 \%$ \\
Divorciado & 5 & $0,0 \%$ \\
Viúvo & 0 & $4,3 \%$ \\
Outro & 2 & \\
\hline Nacionalidade & & $97,9 \%$ \\
\hline Cubanos & & $2,1 \%$ \\
Mauritanianos & $\mathbf{1 0 0 \%}$ \\
\hline Total & $\mathbf{4 7}$ & \\
\hline
\end{tabular}

Fonte: (Baraud, 2016, p. 179-180).

Esses elementos biográficos nos levaram a propor que esses médicos estariam submetidos a diminuição do poder de agir, no nível pessoal, tendo em vista a inserção em contexto pessoal e profissional provisório, transitório, de trabalho fora do país de origem, justificado pela busca de melhoria de vida pessoal e familiar. Quando se comparam médicos em regime de mobilidade profissional exercendo seu trabalho na França e no Brasil (caso dos médicos cubanos aqui em análise), verifica-se que esses últimos são significativamente mais numerosos, ao citar o salário insuficiente no país de origem $\left(X^{2}=4,71 ; \mathrm{p}=0,030\right)$ e a expectativa de melhorar suas condiçôes financeiras $\left(X^{2}=15,3 ; \mathrm{p}=0,000\right)$ como justificativas para a inserção no programa de exercício profissional da medicina fora do país de origem (Baraud, 2016, p. 202). Por outro lado, é preciso ressaltar que a única perspectiva institucionalmente aceita, em relação aos médicos cubanos, é a volta para Cuba. Diferentemente do caso dos médicos em mobilidade na França, os cubanos não podem explorar uma variedade de possibilidades no país de acolhimento para imaginar e elaborar um projeto profissional e de vida privadafamiliar. Essa situação pode gerar sofrimento identitário. Tendo em vista, ainda, que não lhes é facultado falar livremente acerca dessa circunscrição de projeto de vida, os médicos cubanos sofrem importante restrição quanto à elaboração com 
terceiros (notadamente brasileiros) de narrativa sobre o impacto dessa limitação em suas vidas. Eles estão, portanto, confrontados com o obstáculo à expressão, no nível conceitual e cognitivo, das implicações dessa situação. Eles estão livres para falar sobre as consequências afetivas da situação, como a saudade que têm, mas não estão livres para elaborar, em regime de interlocução, acerca da causa da situação, ou seja, da grande implicação do governo cubano na vida pessoal de cada um deles.

Em face das considerações aqui expostas, vale por último mencionar a situação de grande complexidade que resultaria do engajamento de participantes cubanos nas entrevistas programadas, apesar e em confronto (encoberto) com a interdição institucional estabelecida. De fato, muitos dos médicos em questão se disponibilizaram a participar, sob reserva de proteção das respectivas identidades. Além das questôes de ordem ética envolvidas nesse tipo de pesquisa, vale considerar que as narrativas obtidas nesse contexto não seriam desprovidas de valor, mas deveriam passar por trabalho analítico específico. Tal consideração justificou a decisão de não realizar as referidas entrevistas, optando-se pelo uso substitutivo de dados divulgados pela mídia, cruzados com as repostas aos questionários, de forma a construir análise das condições psicológicas do exercício profissional dos médicos cubanos no Brasil, no âmbito do Programa Mais Médicos.

\section{CONSIDERAÇŌES FINAIS: A PESQUISA QUE REFLETE SOBRE SEU FOCO E SIMULTANEAMENTE SOBRE SUAS VICISSITUDES}

Contrariamente à tradição de pesquisa que estabelece um desenho preliminar (design) que guiará sua feitura, garantindo preceitos centrais como aqueles referentes à replicabilidade, estabelecimento claro de variáveis em jogo (dependentes e independentes) e definições operacionais de tais variáveis e procedimentos que lhes são associados, a abordagem clínica, que de fato orienta e dá sentido epistemológico às abordagens psicossociológicas da atividade do trabalho, estabelece como crucial a disponibilidade do pesquisador para o processo contínuo de co-gênese do objeto de pesquisa, ao longo da mesma. Isso significa que não se tem, na partida, de forma predefinida, o foco de recorte pretendido, os objetivos e os caminhos metodológicos de pesquisa. Tal postura, claramente presente nas abordagens experimentalistas da pesquisa científica, não se coaduna com uma abordagem para a qual o conhecimento necessariamente processual de determinado recorte de fenômeno não se circunscreve a ele, tal como a alteridade, inerente aos indivíduos que dele participam e, por extensão, aos indivíduos que pretendem descrevê-lo (nós, pesquisadores envolvidos).

A recusa de autorização da autoridade de supervisão dos médicos cubanos 
vinculados ao programa institucional brasileiro Mais Médicos, vivida inicialmente como fonte de limitação de nosso poder de agir como pesquisadores, e, portanto, fonte de frustração e sofrimento, ensejou, em sua vivência, o refinamento de nossa pretensão inicial a uma abordagem psicossociológica da atividade de trabalho (através do apelo a operadores teóricos oriundos da psicologia histórico-cultural (Vygotski, 2014), filosofia da linguagem (Bakhtine, 1977) e clínica da atividade (Clot, 2010a, 2010b; Clot \& Gollac, 2014). Tal refinamento implicou, num primeiro momento, uma ampliação de nossa própria consciência (Vygotski, 2003) acerca de nosso caminho de pesquisa - mais impregnado dos preceitos de uma prática hegemônica (experimentalista) de pesquisa do que havíamos até então suspeitado. Para além da ampliação e refinamento da descrição da atividade de trabalho dos médicos cubanos operando profissionalmente no Brasil, a vicissitude representada pela recusa aqui aludida possibilitou a ampliação e o refinamento de nossa própria atitude de pesquisa, pela constatação de que a necessária atitude de consideração da co-gênese com a qual pretendemos abordar nossa problemática em psicologia do trabalho deve, necessariamente, referir-se coerentemente ao nosso próprio fazer como pesquisadores. 


\section{REFERÊNCIAS}

Bakhtine, M. (Volochinov, V.N.) (1977). Le Marxisme et la philosophie du langage: essai d'application de la méthode sociologique en linguistique. Paris: Les Éditions du Minuit.

Baraud, M. (2016). Processus identitaires personnels et professionnels et trajectoire migratoire chez des médecins diplômés à l'étranger: une étude exploratoire en France et au Brésil. (Tese de Doutorado), co-tutela Université Lumière Lyon 2, Universidade Federal do Rio Grande do Norte, Lyon (França) -Natal (Brasil).

Barros Neto, N. (2013, 25 agosto). Não somos prisioneiros do regime, dizem médicos cubanos ao chegar à Bahia. Salvador, Folha de São Paulo, Cotidiano. Recuperado a partir de http://www1.folha.uol.com.br/ cotidiano/2013/08/1331912-nao-somos-prisioneiros-do-regime-dizemmedicos-cubanos-ao-chegar-a-bahia.shtml

Bendassolli, P.\& Sobol, L.A.P. (2010). Clinicas do trabalho: novas perspectivas para compreensão do trabalho na atualidade. São Paulo: Atlas.

Brasil. (2017). Programa Mais Medicos. Brasília: Mais Médicos. Recuperado a partir de http://maismedicos.gov.br/

Brito, J. A. (2014). Cuba em transformação: regime político e o contexto da "atualização" do modelo econômico e social". Rebela: Revista Brasileira de Estudos Latino-Americanos 3(2) Recuperado a partir de https://rebela.emnuvens.com. $\mathrm{br} / \mathrm{pc} /$ article/view/136/312

Bruner, J. (2014). Culture et esprit: une féconde incommensurabilité. In: Moro, C., Muller Mirza, N. (Orgs.). Sémiotique, culture et développement psychologique. Villeneuve d'Ascq: Presses Universitaires du Septentrion.

Clot, Y. (2010a). Trabalho e poder de agir. Belo Horizonte: Fabrefactum.

Clot, Y. (2010b). Le travail à coeur: pour en finir avec les risques psychosociaux. Paris: La Découverte.

Clot, Y., Gollac, M. (2014). Le travail peut-il devenir supportable?. Paris: Armand Colin.

Lhuilier, D. (2006). Cliniques du travail. Paris: Érès.

Melgaço (2014, 11 julho). Mais Médicos - Minas Gerais. Diário do Centro do Mundo. [YouTube]. Recuperado a partir de https://www.youtube.com/ watch?v=IHRcDJzpqL0 
Markovà, I. (2000). Amédée or how to get rid of it: social representations from a dialogical perspective. Culture \& Psychology. 6 (4), 419-460.

Markovà, I. (2006). Dialogicidade e representaçôes sociais: as dinâmicas da mente. Petrópolis: Editora Vozes.

Rommetveit, R. (2003). On the role of "a psychology of the second person" in studies of meaning, language and mind. Mind, culture and activity. 10(3), 205-218.

Valsiner, J. (2000). Culture and human development. London: Sage Publications.

Valsiner, J. (2001). Comparative study of human cultural development. Madrid: Fundación Infancia y Aprendizaje.

Valsiner, J. (2014). An invitation to cultural psychology. Los Angeles: Sage Publications.

Veresov, N. (2014). Émotions, perezhivanie et développement culturel: le projet inachevé de Lev Vygostki. In: Moro, C., Muller \& Mirza, N.(Eds), Sémiotique, culture et développement psychologique. Villeneuve d'Ascq: Presses Universitaires du Septentrion.

Vygotski, L. (2003). Conscience, inconscient, émotions. Paris: La Dispute.

Vygotski, L.S. (2014). Histoire du développement des fonctions psychiques supérieures. Paris: La Dispute.

Walter, J. D., Pontes N., Valle. A. (2014, 27 outubro). Cuba e os dois lados do envio de médicos para combater o ebola na África. $D$ W Made for minds. Recuperado a partir de http://tinyurl.com/zjckaeo 